Corrigendum

\title{
Corrigendum to "Moderate Fluid Shear Stress Could Regulate the Cytoskeleton of Nucleus Pulposus and Surrounding Inflammatory Mediators by Activating the FAK-MEK5-ERK5-cFos-AP1 Signaling Pathway"
}

\author{
Dongping Ye $\mathbb{D}$, Weiguo Liang $(\mathbb{D}$, Libing Dai, and Yicun Yao \\ Guangzhou City Red Cross Hospital, The Fourth Affiliated Hospital of Medical College, Jinan University, Guangzhou 510220, China \\ Correspondence should be addressed to Dongping Ye; yedongping927@126.com \\ Received 22 July 2018; Accepted 29 July 2018; Published 27 September 2018 \\ Copyright (C) 2018 Dongping Ye et al. This is an open access article distributed under the Creative Commons Attribution License, \\ which permits unrestricted use, distribution, and reproduction in any medium, provided the original work is properly cited.
}

In the article titled "Moderate Fluid Shear Stress Could Regulate the Cytoskeleton of Nucleus Pulposus and Surrounding Inflammatory Mediators by Activating the FAKMEK5-ERK5-cFos-AP1 Signaling Pathway" [1], a grant number was missing. The Acknowledgments section should be updated as follows:

This project was supported by the fund of Medical and Health Project of Guangzhou City (20151A011013, 20161A011013, 20171A011249, and 20171A011012), the fund of Guangzhou Municipal Science and Technology Bureau (201607010051), the fund of Guangdong Science and Technology Department (2013B021800070), and the Guangdong Provincial Natural Science Fund of China (2015A030313736 and 2018A030310021).

\section{References}

[1] D. Ye, W. Liang, L. Dai, and Y. Yao, "Moderate fluid shear stress could regulate the cytoskeleton of nucleus pulposus and surrounding inflammatory mediators by activating the FAKMEK5-ERK5-cFos-AP1 signaling pathway," Disease Markers, vol. 2018, Article ID 9405738, 9 pages, 2018. 


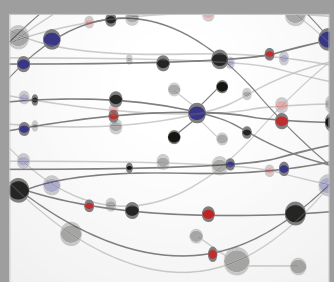

The Scientific World Journal
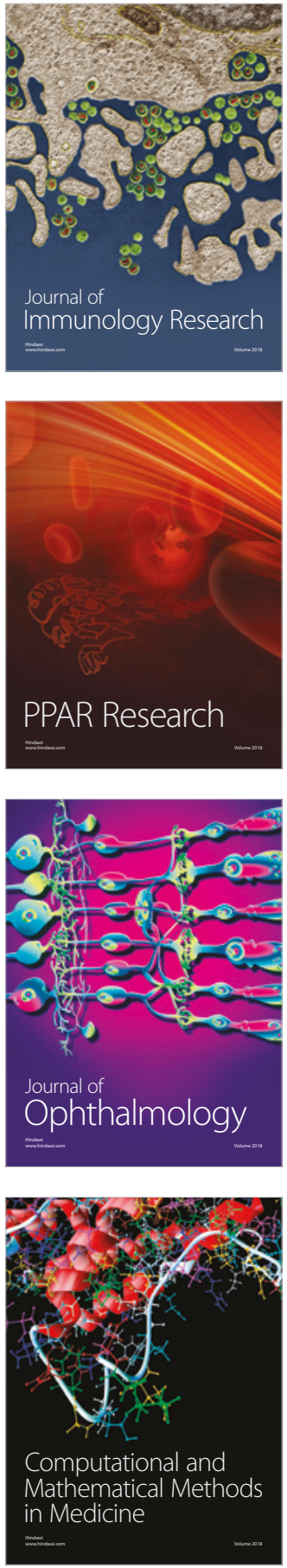

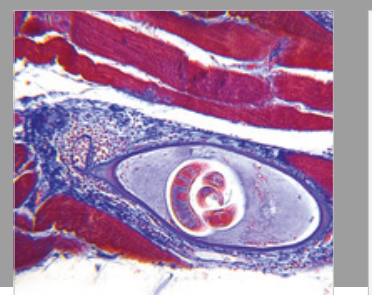

Gastroenterology Research and Practice

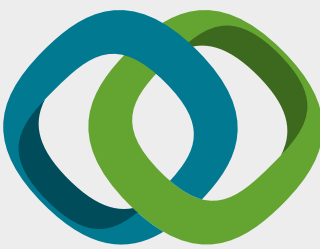

\section{Hindawi}

Submit your manuscripts at

www.hindawi.com
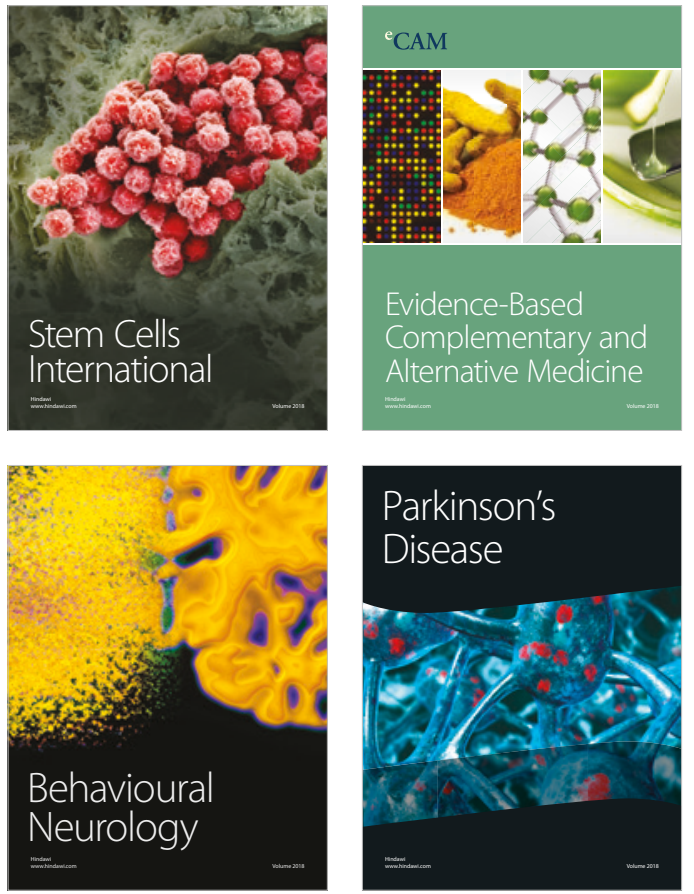

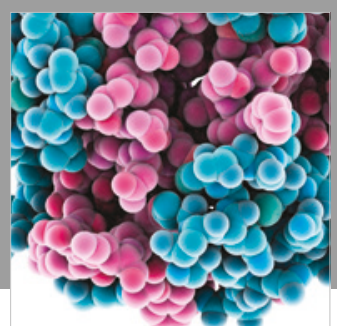

ournal of

Diabetes Research

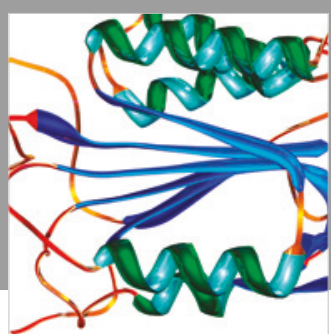

Disease Markers
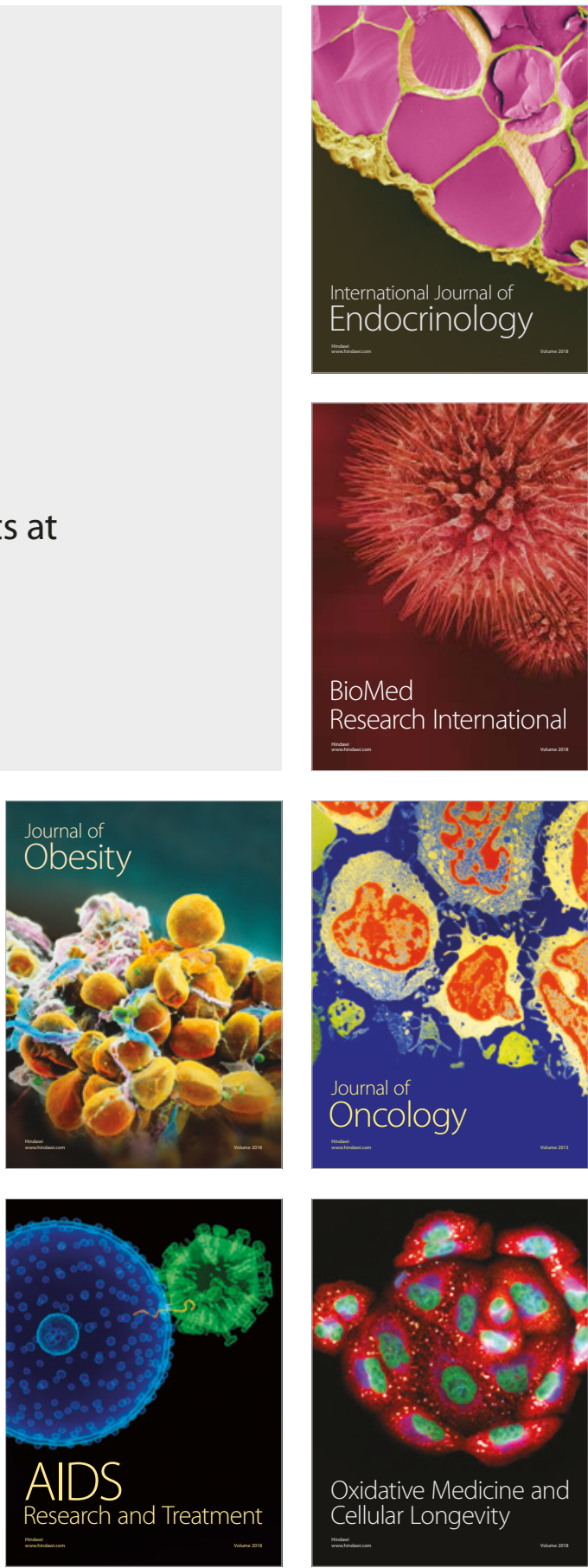